\title{
Synthesis and Applications of Nano Metallic Particles Anchored on a Novel Polymeric Resin
}

\author{
JAYA T. VARKEY \\ Department of Chemistry, St. Teresa's College, Ernakulam, Kochi, Kerala, India, 682035 \\ *Corresponding author E-mail: jayavarkey@yahoo.com \\ http://dx.doi.org/10.13005/ojc/330261
}

(Received: March 18, 2017; Accepted: March 17, 2017)

\begin{abstract}
Nanocatalysis is a rapidly growing research field which involves the use of nanomaterials as catalysts for both homogenous and heterogeneous catalysis. Because of the huge surface area contrasted to bulk matter nanomaterials can function as more efficient catalysts. The main challenge in applying nanoparticles in catalysis is to stabilize and control their size. This problem can be solved by incorporating metal nanoparticles in channels of solid supports. Resin supported metal nanoparticles are popular in many catalytic reactions. Crosslinked polymers are superior because of their ease of filtration and purification. But most of the resins currently using are of having poor reactivity due to their low strength. The support is not acting as a passive solid carrier and the crosslinked polymer is notably influencing the rate of the reactions carried on it. In this review polystyrene crosslinked with 1,6 - hexanediol diacrylate (HDODA) is introduced for nanoparticle supported catalysis. The amount of crosslinking should be accurately adjusted because this has an intense effect on the reactivity of the synthesized polymer. The flexible nature and suitable swelling features of the styrene-HDODA resin will facilitate successful synthesis and the swelling property of this resin is much more compared to the resins commonly used now. The preparation, characterization and catalytic applications of functional polymers are presented.
\end{abstract}

Keywords: polymeric resin, flexible, heterogeneous, metal nanoparticle, catalysis, antimicrobial.

\section{INTRODUCTION}

Since the introduction solid phase synthesis of functional polymers have found extensive applications in organic synthesis ${ }^{1}$. They have been used as reagents, catalysts, in chromatography, in medicine and as substrate carriers 2. One of the important applications of polymer supports is in the field of catalysis ${ }^{3}$. A polymer metal complex is mainly composed of a synthetic polymer and a metal ion. This complexation imparts incorporation of inorganic functions to organic polymer. Immobilizing homogenous metal complexes on polymers increases the complex's catalytic activity, selectivity, reusability and stability of the catalytic species. Various chelating polymeric ligands having different functional moieties attached on numerous supports are reported ${ }^{4,5}$. These polymer supported metal 
complexes show increased catalytic properties than the homogenous metal catalysts. Anchoring of metals on polystyrene supports provides many benefits compared to conventional catalysis. After the completion of the reaction the polymer anchored metal can be retrieved easily by filtration. Since the catalyst is heterogeneous, polluting the products can be prevented. The catalyst can be recycled making this more of environment safe. Selectivity of the catalyst can be achieved by this approach. Numerous supports are available for anchoring metals. Study of various characteristics of polymer assisted reactions revealed the impact of the insoluble support on the reactivity of the attached substrates. Homogenous transition metal catalysts are the most active agents for coupling reactions. But efficient separation and recycling are the major challenges. Heterogeneous catalysis plays a critical role in chemical industry. For this the catalyst should be dispersed on a flexible polymer support for its efficient activity in catalytic processes. This review will focus on the advantages of polymer supported catalysis with emphasis given to the advantages of metal nanoparticle as catalyst.

\section{Merits of Catalysis By Resin supported metal nanoparticles}

The major benefit of adopting polymer supported metals in catalysis is attributed to the simplicity of the process. In the case of crosslinked polymers simple filtration can be used for isolation and washing and the need for complex chromatographic techniques can be eliminated. The catalytic sites are generally present on the surface of metals. Hence nanoparticles which have huge surface area than the bulk matter are promising materials for catalysis ${ }^{6,7}$. Thus the metal nanoparticle can act as the plot for catalysis with increased reactivity. They can also provide assistance in the catalytic process. One significant advantage is that these nanoparticle catalysts can be simply removed and recycled. These types of metallic nanoparticles anchored on polymer supports can be used as catalysts even in gaseous reactions.

\section{the Selection of Polymer Support}

The most important part in resin supported synthesis is rooted on the selection of polymer support. The nature of selected resin plays a pivotal role in the success of the synthesis. A functionalized resin is a cross linked polymer where functional groups are attached through a chemical bond and these groups can be regents, scavengers or catalysts. Development of polymer supports which swell in both polar and non-polar solvents has been a challenge to

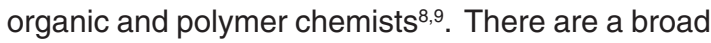
array of polymer supports available into which metal nanoparticles can be anchored but the concept of optimum hydrophobic-hydrophilc balance and the flexibility of the supports is the deceive factor for the effective reactivity. Despite the many advantages of widely used styrene-DVB (divinyl benzene) resin (Figure 1) its low swelling and solvation in polar organic solvents, and poor loading attributed lower accessibility by reagents ${ }^{10,13}$.

In addition to PS-DVB resin a number of polymers are used in the development of polymer<smiles>C=Cc1ccc(C=C)cc1</smiles>

Fig. 1: DVB-crosslinked polystyrene 
supported catalysts and reagents. The important ones are PEG crosslinked PS ${ }^{14,15}$, Janda gel ${ }^{16}$, Tenta gel ${ }^{17}$ and polyacrylamides ${ }^{18}$. Various organic polymer supports used were extensively reviewed ${ }^{19,20}$. The matter of slow reactions and poor yield could be avoided by proper choice of support and reaction conditions. The general assumption is that the polymeric support is doing only the role of a carrier. But several studies associated with polymer based reactions have shown that the insoluble polymeric resin does have an active impact on the substrates attached to it. An ideal support should have a perfect hydrophobic-hydrophilic equilibrium. It is proposed to synthesize polystyrene cross-linked with 1 , 6-hexanediol diacrylate (Figure 2) ${ }^{21}$, which can be suitably functionalized to use as the solid support (Figure 3). The flexibility of the HDODA crosslinker and the better swelling properties of styrene-HDODA crosslinked polymer are proved to be much better than Merrifield resin and this will facilitate

the catalytic applications ${ }^{22}$. Another important component of this support is its moderate hydrophilicity due to acrylic groups. The scanning electron micrographs (SEM) (Figure 4 ( $a$ \& b) revealed the surface characteristics of the resin surface before
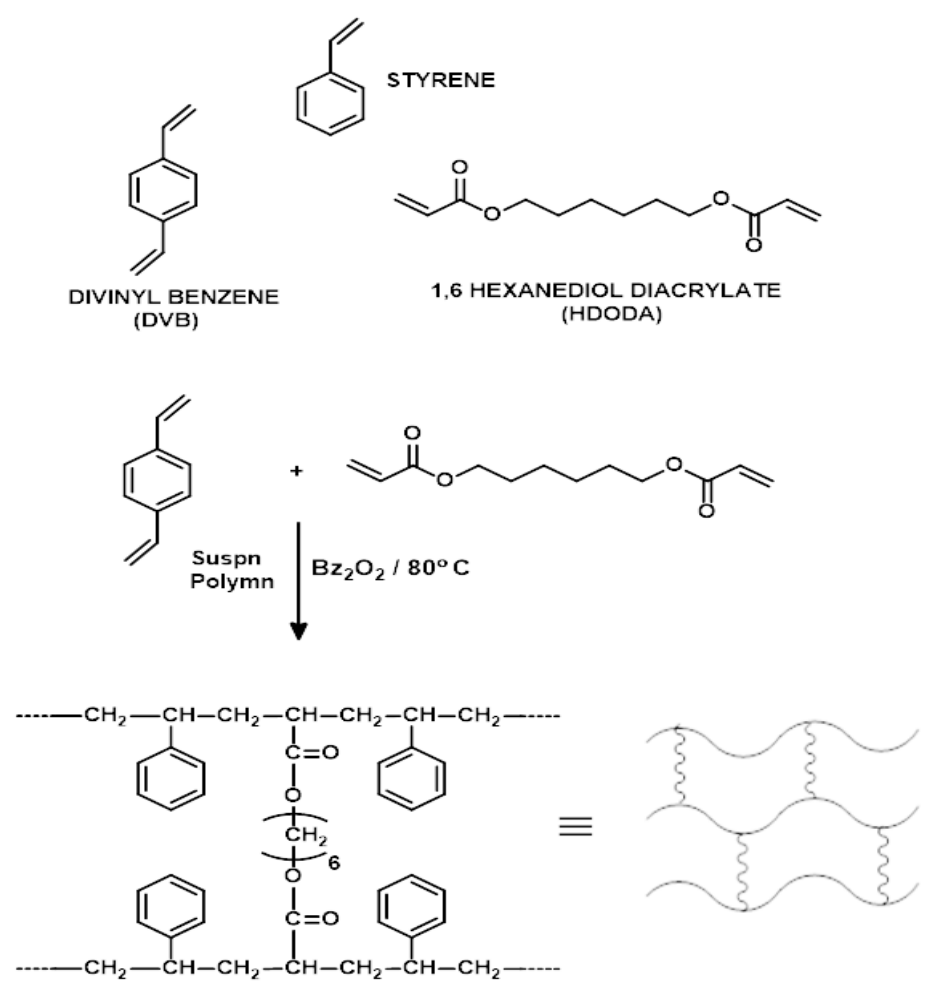

Fig. 2: PS- HDODA resin

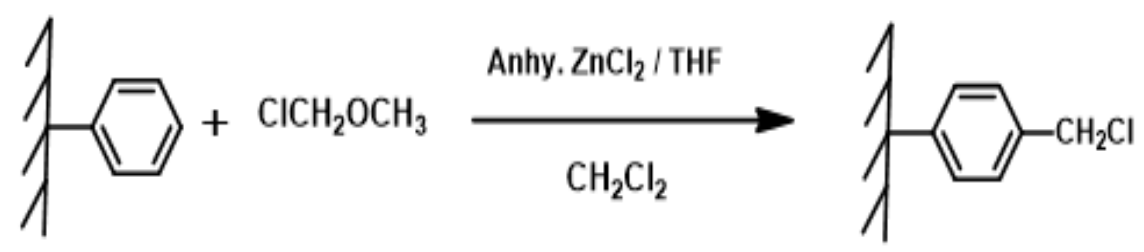

Fig. 3: Chloromethylation of HDODA-crosslinked polystyrene resin 
and after functionalisation. The .smooth resin surface became rough after chloromethylation

\section{Metal Nanoparticle Catalysts}

These catalysts are made up of nanomaterials and are of heterogeneous in nature. Because of the large outer area there are many reactive sites on the surface. Thus numerous catalytic reactions can take place simultaneously and thus the rate of catalytic action is increased. Moreover these types of catalysts can be easily isolated and reused with increased catalytic activity than their bulk analogues ${ }^{23,25}$. They can perform two distinct jobs in catalytic processes: can assist in catalytic operations or can act as the location for these processes. One important condition is that the reaction process should not affect the stability of nanoparticles.

The strength of nanoparticles involved in the reactions is the most important aspect because they have a propensity to aggregate. This agglomeration could result in gradual decrease in catalytic activity. In addition complete separation and reuse of these nanoparticles after each operation can be very difficult. A successful approach to block this aggregation and to make smooth functioning of the metallic nanoparticles is to anchor them on suitable solid supports ${ }^{26}$.

The main challenge in nanoparticles synthesis and their application in catalysis is a necessity to stabilize and control their size. This problem can be solved by incorporating metal nanoparticles in channels of solid supports or

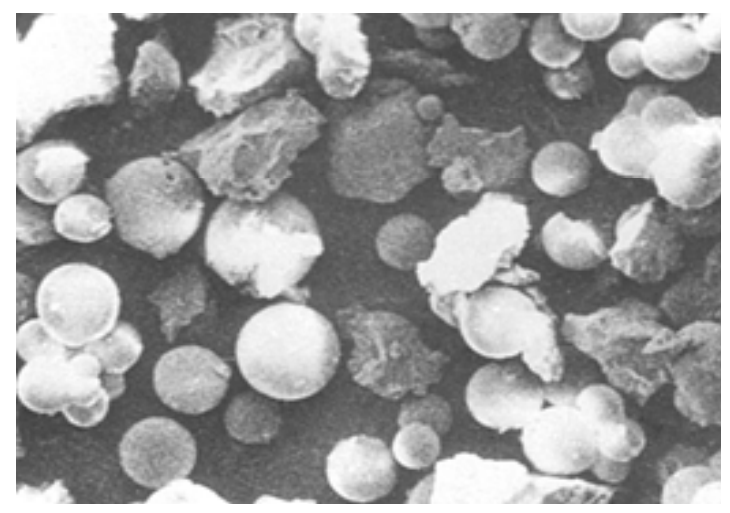

a stabilizing them with organic low-molecular and macromolecular ligands. Linear or cross linked polymers can be used as supports for metal nanoparticles. Cross linked polymers are more useful because of their ease of filtration and purification. These polymers consist of infinite network in which linear chains are interconnected. The poor physical and chemical stability is the main disadvantage for most of the support materials used nowadays.

\section{Coordination With Metal Nanoparticles}

The percentage of cross linking agent plays a crucial role on the morphology of the resin. So it is utmost important to control the amount of crosslinking agent when carrying out polymerization. Functionalisation of the crosslinked PS -HDODA support can be carried out using chloromethylation or sulphonation. The metal is introduced into the functionalized resin by using suitable metal precursors. Depending upon the nature of the functional groups ion exchange process can be used to incorporate metal cations ${ }^{6,7}$. In this approach the choice of solvents should be carefully controlled to avoid reduction of metal.

\section{Applications of Polymer Supported Metal Complexes Catalysis}

Nanostructured metal particles dispersed on functional polymers provide a large class of useful catalysts ${ }^{26}$. Many hydrocarbon reactions like hydrogenation, hydrosilylation and hydrofromylation can be carried out by these polymer supported complexes. Their applications also include removal

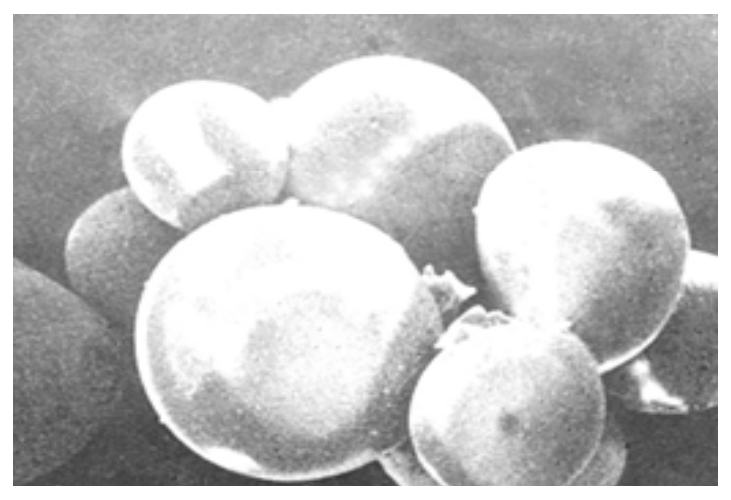

a

Fig. 4: SEM images of (a) PS-HDODA beads (b) chloromethylated resin 
of hydrogen from water, nitrates from drinking water and in coupling and substitution reactions ${ }^{27,28}$.

\section{Antimicrobial activity}

Metals and their nanoparticles exhibit potential antimicrobial property [29]. The bactericidal effect of metal nanoparticles has been assigned to their small size. The biocidal activity of these metal nanoparticles can be further enhanced by incorporating them into functionalized polymers. There are several studies dealing with silver and copper nanoparticles incorporated on polymer matrices $[30,31]$ but the flexible nature of PSHDODA support is not much exploited for this pupose.

\section{CONCLUSION}

Interest in polymer supported metal complexes has increased recently due to its industrial demands. With the evolution in nanotechnology supported metal nano particles have found tremendous applications in various fields. By the immobilization of homogenous catalysts on functional organic polymers their efficiency could be increased. The properties of the resin support play an active role in this heterogeneous catalysis. By suitably distributing metal nano particles into synthetic polymers many of the problems associated with them could overcome without disturbing the parent properties of nanoparticles. New supports with more swelling and solvation need to be investigated to improve the potentialities of these resin supported catalysts. Presence of flexible and slightly hydrophilic cross linking agent makes the PS-HDODA support suitable for applications of metal nano particles dispersed on it. These resins can be characterized by IR, solid state ${ }^{13} \mathrm{C} C P / M A S ~ N M R$ spectroscopy and SEM. The participation of reusable resin bound catalyst is a clear example for green chemistry.

\section{ACKNOWLEDGEMENT}

The author would like to thank University Grants Commission, New Delhi, India for financial assistance in the form of UGC research award.

\section{REFERENCES}

1. Akelah, A. ; Sherrington D. C. Recent developments in the application of functionalized polymers in organic synthesis. Polymer, 1983, 24, 1369-1386.

2. Clapham, B.; Reger,T, S.; Janda K. D. Polymer-supported catalysis in synthetic organic chemistry. Tetrahedron, 2001, 57(22), 4637-4662.

3. Leadbeater N. E; Marco, M. Preparation of Polymer-Supported Ligands and Metal Complexes for Use in Catalysis. Chem. Rev., 2002, 102 (10), 3217-3274.

4. Nishide, E, T. Advances in Polymer Sciences, Springer-Verlag, Berlin, 24, 1977.

5. Wohrle, D. Advances in Polymer Sciences, Springer-Verlag, Berlin, 50, 1983.

6. Kralik, M,; Corain, B.; Zecca, M. Catalysis by metal nanoparticles supported on functional polymers. Chem. Papers, 2000, 54(4), 254264.

7. Kralik, M.; Biffis, A. Catalysis by metal nanoparticles supported on functional organic polymers. Journal of Molecular Catalysis A: Chemical, 2001, 177, 113-138.

8. Small P.W.; Sherrington D. C. Preparation, structure and morphology of polymer support. J. Chem. Soc. Chem. Commun., 1989, 21, 1589-1591.

9. Auzanneau, F. I.; Meldal, M.; Bock, K. Synthesis, characterization and biocompatibility of PEGA resins. J. Peptide Sci., 1995, 1, 31-44.

10. Shuttleworth, S. J.; Allin, S. M.; Wilson R, D.; Nasturica, C. Functionalised polymers in organic chemistry, part 2. Synthesis, 2000, 8, 1035-1074.

11. Clapham, B.; Reger, T. S.; Janda, K. D. Polymer supported catalysis in synthetic organic chemistry. Tetrahedron, 2001, 57, 4637-4662.

12. Benaglia, M; Puglisi, A.; Cozzi, F. Polymersupported organic catalysts. Chem. Rev., 
2003, 103, 3401-3429.

13. Benaglia, M. Recoverable and recyclable chiral organic catalyst. New J. Chem., 2006, 11, 1525-1533.

14. Luo, J,; Pardin, C.; Lubell, W. D.; Zhu, X. X. Poly(vinyl alcohol)-graft-poly(ethylene glycol) resins and their use in solid-phase synthesis and supported TEMPO catalysis. Chem. Commun., 2007, 21, 2136-2138.

15. Renil, M.; Nagaraj, R.; Pillai V. N.R. Gel-phase peptide synthesis on a new high-capacity tetraethyleneglycol diacrylate-crosslinked polystyrene support: Synthesis of pardaxin 16-33. Tetrahedron, 1994, 50, 6681-6688.

16. Spanka, C; Clapham, B.; Janda, K. D. Preparation of new microgel polymers and their application as supports in organic synthesis. J Org Chem., 2002, 67(9), 30453050.

17. Rapp, w. In combinatorial peptide and nonpeptide libraries, Jung, G, Ed. ; VCH, New York, p 425, 1996.

18. Skaria, S.; Rajan, R.; Ponrathnam, S. Redox polymerization of acrylamide by polymer supported vanadium (V) ion. Polymer, 1997, 38(7), 1699-1704.

19. Lu, J.; Toy, P. H. Organic Polymer Supports for Synthesis and for Reagent and Catalyst Immobilization. Chem. Rev. 2009, 109, 815-838.

20. Bergbreiter, DE. Polymer supports in organic catalysis and synthesis. Curr. Opin. Drug Discov. Devel. 2001, 4(6), 736-44.

21. Varkey, J. T.; Pillai, V. N. R. Synthesis of thioredoxin partial sequences on 1, 6-hexanediol diacrylate (HDODA) crosslinked polystyrene resin. Journal of peptide research, 1998, 51, 49-54.

22. Varkey, J.T.; Pillai, V.N. R. Merrifield resin and newly developed 1, 6-hexanediol diacrylate resin for solid phase peptide synthesis. A comparative study. Journal of applied polymer science, 1999, 71, 1933-39.

23. Rao, C.N. R.; Kulkarni, G. U.; Thomas, P. J.; Edwards, P. P. Metal nanoparticles and their assemblies. Chem. Soc. Rev., 2000, 29, 27-35.

24. Shiju, N, R.; Guliants, V, V. Recent developments in catalysis using nanostructured materials. Applied Catalysis A: General, 2009, 356, 1-17.

25. Singh, S. B.; Tandon, P. K. Catalysis: A brief review on nano-catalyst. Journal of energy and chemical engineering, 2014, 2, 3, 106115.

26. Corain, B.; Zecca, M.; Canton, P.; Centomo, P. Synthesis and catalytic activity of metal nanoclusters inside functional resins: an endeavour lasting 15 years. Phil. Trans. $R$. Soc. A, 2010, 368, 1495-1507.

27. Karimi, B.; Behzadnia, H.; Farhangi, E.; Jafari, E.; Zamani, A. Recent application of polymer supported metal nanoparticles in Heck, Suzuki and Sonogashira coupling reactions. Current Organic Synthesis, 2010, 7, 543-567.

28. Castro, I. U.; Sherrington, D. C.; Fortuny, A.; Fabregat, A.; Stuber, F.; Font, J.; Bengoa, C. Synthesis of polymer-supported copper complexes and their evaluation in catalytic phenol oxidation. Catalysis Today, 2010, 157, 66-70.

29. Rana, S.; Kalaichelvan, P. T. Antibacterial activities of metal nanoparticles. Advanced Biotech. 2011, 11(2), 21-23.

30. Rawashdeh, R.; Haik, Y. Antibacterial mechanisms of metallic nanoparticles: A review. Dynamic Biochemistry, Process Biotechnology and Molecular Biology, 2009, 3, 2,12-20.

31. Palza, H. Antimicrobial polymers with metal nanoparticles. Int. J. Mol. Sci., 2015, 16, 209, 9-2116. 